\title{
Carbon Stock Assessment at a Regional Level, on Pure and Mixed Stands, Based on Inventory Data: A Case Study ${ }^{*}$
}

\author{
Teresa Fidalgo Fonseca, Carlos Pacheco Marques \\ Department of Forest Science and Landscape Architecture, University of Trás-os-Montes e Alto Douro, Vila Real, Portugal. \\ Email: tfonseca@utad.pt \\ Received July $4^{\text {th }}, 2011$; revised July $19^{\text {th }}, 2011$; accepted July $22^{\text {th }}, 2011$.
}

\begin{abstract}
Following Kyoto protocol considerable research efforts have been made on carbon sequestration assessment of forest stands. The aim of the present study was to test the adequacy of two documented methodologies to obtain stand level dry biomass and carbon estimates, considering its application at regional scale, for pure and mixed stands. The procedures refer to: 1) the estimation of dry biomass through the use of tree biomass models and 2) the use of biomass conversion and expansion factors (BCEF) to convert stem volume raw data into aboveground dry biomass. Using the dry biomass values, carbon stock is easily estimated using conversion factors, being 0.50 a generally accepted value.

Case study reports to a pilot zone with pure and mixed stands of Eucalyptus globulus Labill and Pinus pinaster Ait. in Portugal. Procedure 1) gives the most reliable results when the database includes detailed information at tree level, both for pure or mixed stands. When individual tree data is not accessible, option 2) is an interesting option to consider for pure stands. The use of BCEF-based procedure in mixed stands is not advocated at least when considering the direct use of a single conversion factor, as the value of the factor strongly depends on the forest composition.
\end{abstract}

Keywords: Biomass, BCEF, Greenhouse Inventory, Carbon Monitoring

\section{Introduction}

The assessment of carbon in the forest areas is a timely subject because carbon stock is an important criterion of Sustainable Forest Management and at the same time it is required for greenhouse gas inventories needed in the LULUCF sector (Land Use, Land Use Change and Forestry) for the United Nations Framework Convention on Climate Change (UNFCCC) reporting.

Two major methodologies have been proposed and accepted for use to assess the carbon stock of the tree component using ground level inventory data. One is based on tree biomass and carbon concentration in the biomass ( $\mathrm{g}$ of carbon per $\mathrm{g}$ of biomass). Under this approach, tree dry biomass is estimated from tree characteristics using allometric equations that relate tree biomass with tree size (usually, diameter or diameter and height). For general forest inventory purposes this approach seems to be the most reliable provided that there are biomass models available for the inventoried species. This seems not to be of great concern as there is a wealth of literature about the subject for both whole tree biomass and the biomass of different components. Just to mention some biomes, Zianis et al. (2005) present a database on biomass equations for various tree species growing in Europe. A compilation for North-American and for Canadian tree species can be found in Ter-Mikaelian and Korzukhin (1997) and Lambert et al. (2005), respectively.

However, it is not unusual a lack of recorded values on individual tree diameters, namely for evaluations made at sub-regional and at regional scale. In such cases, alternative methods based on inventory data need to be considered. One of the most practical approaches makes use of stand volume data (see Brown et al. 1989; Brown 1997). Biomass (B) is estimated from volume ( $v$ ) data using the general formula $B=\rho E v$, where $\rho$ represents wood density and $E$ goes for an expansion factor used to expand values from one tree component, usually the stem, to other tree components (e.g. crown) to get aboveground estimates. Two main options are offered under this approach. One option, not discussed here, refers to the use of the individual values of tree volume and wood density. Dry mass values are calculated using the volume of the trees (measured or estimated) and the wood density values for the species (a large database of wood density values is available from the World Agroforestry Centre website). This is a helpful option when is required a roughly estimate of carbon and there is no information of proper biomass equations for the existing species.

Other option requires the use of biomass conversion (BCF) and biomass expansion factors (BEF). Those combined factors (BCEF) allow converting stem volume raw data per unit area into stem dry biomass and then to expand stem biomass values to other components of the trees, such as crown and roots. This method is one of the methods currently suggested in the Intergovernmental Panel on Climate Change IPCC guidelines based on National Forest Inventories for estimating biomass gains and losses (IPCC 2006, 4.11). The approach presents a great value for monitoring purposes using data from past forest inventories. Criticism has been made to the use of this approach: biomass factors used are, in most cases, not representative, because they are based on local studies (Somogy et al. 2007); forest inventories often report volumes to different standards, e.g., to minimum diameters greater than $10 \mathrm{~cm}$, thus particular care is needed to unify the volume data (Brown, 1997), this being quite relevant either for monitoring as well as for comparison purposes between regions or countries.

Using the dry biomass values, carbon stock of a tree or of a 
stand is easily estimated for either method, using conversion factors. The value of 0.50 is the most usual and generally accepted, even if the conversion is not completely accurate.

Additional information and reviews on methodologies for biomass assessment and carbon inventory methods can be found in Brown (1997), Ketterings et al. (2001), Lindner and Karjalainen (2007) and Somogy et al. (2008).

The objective of this article is to summarize the results obtained for a pilot zone, with pure and mixed forest stands. Remarks are pointed out along presentation of the two tested methodologies to provide guidance in selecting the best approach to use when evaluating carbon stocks for sustainable forest management at regional level.

\section{Material and Methods}

\section{Case Study}

Under EU funded Project INTERREG III-B FORSEE, carbon stock evaluation from biomass equations and from BCEF approaches were tested for application at regional level, in the Atlantic region. The objective was to assess for relevance and feasibility of the evaluation methods. Case study reports to the essays made under the research project at the pilot region of Sousa Valley, in North Portugal. This region has a total area of 77620 hectares. Sampling area refers to an area of 3384.9 ha on pure and mixed stands of Eucalyptus globulus Labill and Pinus pinaster Aiton. The most represented forest stratum refers to pure stands of eucalyptus $(E c)$ with an area of 1801.0 ha, followed by the mixed stands of eucalyptus and pine $(E c \times P p)$ with 1314.5 ha. Maritime pine pure stands $(P p)$ occupy 269.4 ha.

Data refers to 35 plots, sampled in 2005 according to a systematic grid that was previously superimposed in the study area for inventory purposes of the forest existences in 2003. Plots were circular in size with an area of $500 \mathrm{~m}^{2}$.

According to the layout of the systematic grid, 23 plots located on Ec stratum; 4 plots were placed on stratum $P p$ and 8 plots were located on $E c \times P p$ stratum, of mixed stands of eucalyptus and pine.

The age of the trees ranged from 4 to 14 years for the eucalyptus and from 4 to 54 years for the pine species. An unevenaged structure was reported for the mixed stands and in a number of pure stands for either species.

At each plot, all living trees with $1.30 \mathrm{~m}$ of height were numerated and classified by species and tree status. Diameter at breast height $(d)$ of the living trees were measured with a calliper and the height $(h)$ of a sample of those trees were measured with a Vertex hypsometer. Other species that were recorded in the pure and the mixed stands belong to the Quercus spp gender. A summary of the stand data is presented in Table 1.

\section{Application and Reliability}

\section{Estimation of Dry Biomass through Tree Biomass Models - Compilation Method}

For the two major inventoried tree species, allometric models for the aboveground dry biomass were available for use (Fabião, 1986 and Lopes, 2005). The models use as explanatory variable the diameter at breast height over bark and give estimates of tree aerial biomass per component (stem and crown).

Total aboveground dry mass per plot was obtained by adding
Table 1.

Characterization of the stand variables (mean $\pm s d$ ) for each forest stratum.

\begin{tabular}{cccc}
\hline \multirow{2}{*}{ Variable } & \multicolumn{3}{c}{ Stratum } \\
\cline { 2 - 4 } & Eucalyptus & Pine & Mixed \\
\hline Number of trees $\left(\mathrm{n} . ~ h a^{-1}\right)$ & $1278 \pm 411$ & $690 \pm 329$ & $630 \pm 269$ \\
Basal area $\left(\mathrm{m}^{2} \cdot \mathrm{ha}^{-1}\right)$ & $15.9 \pm 6.0$ & $17.0 \pm 8.1$ & $18.5 \pm 11.4$ \\
Mean diameter $(\mathrm{cm})$ & $12.6 \pm 2.4$ & $17.4 \pm 0.7$ & $18.5 \pm 4.9$ \\
Volume $\left(\mathrm{m}^{3} \cdot \mathrm{ha}^{-1}\right)$ & $120.2 \pm 58.6$ & $122.1 \pm 101.7$ & $137.0 \pm 106.7$ \\
\hline
\end{tabular}

the dry mass of the inventoried single trees. Individual stratum estimates of the mean, denoted as $\bar{B}_{j}$, and of the variance of biomass among plots were calculated using the data of the plots measured in that stratum. Variance of the mean in stratum $j$, was evaluated as,

$$
s_{\bar{B}_{j}}^{2} \approx\left(s_{j}^{2} / n_{j}\right) \times\left(1-n_{j} / N_{j}\right)
$$

where $N_{j}$ refers to the stratum size.

\section{Use of BCEFs to Convert Stem Volume Raw Data into Aboveground Dry Biomass - BCEF Method}

Carbon stock evaluation on forested areas, for which dendrometric data expressed in volume units is available, can be indirectly assessed throughout conversion factors. The method requires the use of consistent factors to convert volume data into dry mass. Depending on which tree compartment is being assessed it may be necessary to expand the converted values to other tree parts. In Portugal, volume data usually refers to stem, hence the factors to apply should make the conversion of stem volume to dry biomass of the stem (implicitly through the density value of wood) and then to expand the estimates of stem biomass to the aboveground components, that is, stem component plus tree crown.

According to the evaluation protocols defined in FORSEE, two variants were proposed for testing. In variant 1 , the estimation of biomass per stratum $j\left(B_{j}\right)$ is made through the multiplication of the volume of the stratum $\left(V_{j}\right)$ by a BCEF value. That is,

$$
B_{j}=V_{j} \times \mathrm{BCEF}
$$

Total biomass is obtained by adding the dry mass values (2) for all strata.

In variant 2 , the data processing requires, at a first step, the estimation of the volume of each tree per plot using a volume equation. Volume per plot is obtained by adding the individual volumes. Plot biomass is then estimated by multiplying plot volume by the $\mathrm{BCEF}(3)$.

$$
B_{i}=V_{i} \times \mathrm{BCEF}
$$

Total biomass per stratum is estimated as the product of the average value of biomass times the stratum area. The sum for all strata provides the estimate of the total biomass.

A prior analysis shown the proposed calculations could present problems, namely for the mixed stands. Hence, a new modification that generalizes the employment of the BCEFbased procedure was proposed for testing by the authors. This new variant, hereafter called variant 3 , requires that the data processing is made at a lower scale level and by species. Briefly, it is completed as follows:

- Compile proper volume equations and BCEF values for each of the inventoried species in the region;

- Using the raw inventory data on tree size, estimate indi- 
vidual volumes;

- Estimate plot volume per specie $\left(V_{i}\right)$ as the sum of individual volumes;

- Estimate plot biomass $\left(B_{i}\right)$ by multiplying plot volume per species with the respective species BCEF value $\left(B C E F_{i}\right)$,

$$
B_{i}=\sum_{i=1}^{\text {Species }} V_{i} \times B C E F_{i}
$$

- Estimate the mean and the variance of the mean per stratum (see method 1).

- Aggregate the strata values using the appropriate formulas for stratified populations.

Volume calculation

For each of the three BCEF-approach variants, the tree volume of the major species was estimated using a local volume equation, developed for the study area (5). Available data base comprised 333 observations of stem volume (outside bark, from base to tip) evaluated on sample standing trees with a Bitterlich relaskop.

$$
v=(0.000458-0.000399 S) d^{2.122+0.574 S}
$$

In Equation $5, v$ refers to stem volume in $\mathrm{m}^{3}, S$ is a binary variable for coding species, being 0 for eucalyptus and 1 for maritime pine; and $d$ is already defined. The standard error $\left(s_{y x}\right)$ of this model is $.049 \mathrm{~m}^{3}$.

For the other species inventoried in the sampled plots, stem volume was directly assessed, with the Bitterlich relaskop. Total stem volume per plot was obtained by adding the volume of the single trees.

BCEF values

The general $B C E F$ values proposed for testing under the FORSEE project were the ones provided by the official agency of Environment: $0.70 \mathrm{t} . \mathrm{m}^{-3}$ for E. globulus and $0.78 \mathrm{t} . \mathrm{m}^{-3}$ for $P$. pinaster. Values refer to the ratio of aboveground biomass (ton) to the stem volume $\left(\mathrm{m}^{-3}\right)$.

Calculations were made also using the factors evaluated for eucalyptus and pine species in the pilot region (mean $\pm \mathrm{sd}$ ): $0.63 \pm 0.07$ and $0.54 \pm 0.05$, respectively, using the available data set of volume and biomass estimates for the sampled plots in the pure stands.

For the mixed stands there are no published values, as the value depends on the species composition and varies with the occupancy of the species.

\section{Overall Estimates of Biomass and of Carbon}

Knowing the confidence interval is as critical a knowledge as the average estimates. Once estimates of individual stratum parameters (mean and standard error) have been computed, an estimate of biomass for all three strata combined is calculated using the formula for the systematic stratified design.

The mean dry biomass for the $M$ strata, denoted $\bar{B}$, and the standard error, denoted by $s_{\bar{B}}$, are given in Equations 6 and 7, respectively.

$$
\begin{array}{r}
\bar{B}=\sum_{j=1}^{M} P_{j} \bar{B} \\
S_{\bar{B}}=\sqrt{\sum_{j=1}^{M} P_{j}^{2} s_{\bar{B}_{j}}^{2}}
\end{array}
$$

In Equations 6 and 7, $P_{j}$ refers to the proportion of stratum $j$
Table 2.

Carbon estimates using the biomass compilation and the BCEF method.

\begin{tabular}{cccc}
\hline \multirow{2}{*}{ Carbon } & $\begin{array}{c}\text { Compilation } \\
\text { method }\end{array}$ & \multicolumn{2}{c}{ BCEF values-variant 3} \\
\cline { 3 - 4 } & 43 & 46 & $\begin{array}{c}\text { Ec: } 0.63 ; \\
\text { Pp: } 0.54\end{array}$ \\
\hline $\begin{array}{c}\text { Mean } \\
\left.\text { (ton. ha }{ }^{-1}\right)\end{array}$ & 47 & 26 & 39 \\
$\begin{array}{c}\text { SE }(\%) \\
\mathrm{CI}^{*} \text { for the } \\
\text { total (ton) }\end{array}$ & $\begin{array}{c}145500 \pm \\
54000\end{array}$ & $158000 \pm 40500$ & $131500 \pm 33500$ \\
\hline
\end{tabular}

*. Confidence Interval ( 0.95 level of confidence)

with $P_{E c}=0.53 ; P_{P p}=0.08$ and $P_{E c \times P p}=0.39$.

The sampling error is given by,

$$
\mathrm{SE}=s_{\bar{B}} t
$$

In stratified layouts which are not proportional to the stratum area it is not possible to determine rigorously the number of degrees of freedom $(d f)$ for the Student's t-distribution. According to the formula proposed by Satterthwaite (1946) a value of $10 d f$ was estimated, with a corresponding $t$ value of 2.228 at $p$ equal to 0.05 .

Calculations of dry mass were made for each of the tested approaches. A factor of 0.50 was employed to convert the mass estimates in carbon stock values.

\section{Results and Discussion}

The analysis of the biomass and carbon stock evaluation methods shows that the compilation method based on individual tree biomass evaluation is generally applicable, allowing its use in pure and mixed stands.

The method that uses volume data and BCEF values proved to be inadequate for a widespread use, at least as stated in both variants approved for evaluation in the FORSEE project. In fact, variant 1 of BCEF-based method was immediately excluded as it cannot be directly used on mixed stands. Variant 2 of BCEFbased method is also not satisfactory as it assumes a unique BCEF value for the plot, (see 3) independently of the plot composition. The exception would be to have a common BCEF value for all inventoried species, which is not likely to occur in nature. The problem could be overridden by knowing the species contribution in each plot and then to use ratios. Variant 3 is a generalized alternative that avoids the use of ratios while accounting for the stand composition, in mixed stands.

Table 2 presents the essential results on carbon stocking for the study area using the biomass compilation method and the new version of the BCEF-based method. Depending on the method used and on the BCEF values adopted (general or regional ones) estimates of dry biomass in the pilot region vary from 78 to 92 tons per hectare, corresponding to variations of carbon stocks estimates of $17.9 \%$. The sampling error ranges from 25 to $37 \%$ which might be considered high for an assessment at regional level. This is due to the forest heterogeneity and to the sample size.

Although confidence intervals overlap for the alternative estimation procedures, the results clearly point out, that different data processing could led to quite different estimates of biomass and therefore to different evaluations of carbon storage. The under- or the upper- estimation is of concern as it can have 
great effects on national policies with respect to compliance with the Kyoto protocol.

A cautionary note should also be made about the accuracy of the estimates. At a first analysis it would appear that the biomass compilation method produce a greater uncertainty than the BCEF-based ones. This may not be true as the use of a constant $\mathrm{BCEF}$ reduces the variability in the biomass plot estimates, namely on mixed stands stratum. The wider confidence interval computed with the former comes from a greater individual variability in the biomass plot estimates.

\section{Conclusions}

The present study focused on the use of two widely divulgated approaches for dry biomass and carbon stock assessment in forest areas. The key point here was to evaluate the appropriateness of the use at a regional scale, using sample inventory data. Results showed that the approach based on biomass estimation with equations (compilation method) is of easy data handling and is compatible with forest inventories at local, regional or national level. When biomass equations are not available, carbon stock might be evaluated indirectly from volume estimates. The use of BCEFs appears as a promising approach as they can be readily used with existing forest volume inventory data to obtain estimates of biomass stocks. Remarks are made concerning data processing using BCEF-based approaches. Estimating the biomass of mixed stands using this approach is not always feasible as some of the existing variants of calculation are appropriate to apply just to pure stands. A modification of BCEF-based method that accounts for mixed stands is presented. The new modification also applies to pure stands with woody biomass from dominated species. In order to get consistent estimates of dry biomass, the BCEF-based approach needs, however, to be reliable. The example provided in this paper clearly shows how the carbon stock estimates depend on the value of the factor used. Biomass conversion and expansion factors obtained from different sources might give quite diverse estimates of carbon stocking. It is necessary to do more research in this field, including the analysis of the factors variation with stand characteristics.

The statements made for the pilot zone can be extended to other regions and are expected to assist for enhancement of carbon-related assessment issues, including emissions from forest fires or removals from forest harvesting, at the regional level, and for supporting monitoring and reporting programs requiring information about carbon, such as case of the REDD + program.

\section{Acknowledgements}

T.F. Fonseca thanks to Prof. Américo Mendes from the Portuguese Catholic University for his invitation to participate in the FORSEE Project.

\section{References}

Brown, S., (1997). Estimating biomass and biomass change of tropical forests: A primer. FAO Forestry Paper 134.

Brown, S., Gillespie, A. J. R., \& Lugo, A. E. (1989). Biomass estimation methods for tropical forests with applications to forest inventory data. Forest Science, 35, 881-902.

Fabião, A. M., (1986). Contribuição para o estudo da dinâmica da biomassa e da produtividade primária líquida em eucaliptais-região litoral do centro de Portugal. Ph. D. Thesis, Lisbon: Universidade Técnica de Lisboa.

IPCC, 2006. Guidelines for national greenhouse gas inventories. Volume 4: agriculture, forestry and other land use. URL (last checked 29 June 2011)

http://www.ipcc-nggip.iges.or.jp/public/2006gl/pdf/4_Volume4/V4 04 Ch4 Forest Land.pdf

Ketterings, Q. M., Coe, R., van Noordwijk, M., Ambagau, Y., \& Palm, C. A. (2001). Reducing uncertainty in the use of allometric biomass equations for predicting above-ground tree biomass in mixed secondary forests. Forest Ecology and Management, 146, 199-209.

Lambert, M. C., Ung, C. H., \& Raulier, F. (2005). Canadian national tree aboveground biomass equations. Canadian Journal of Forest Research, 35, 1996-2018.

Lindner, M., \& Karjalainen, T. (2007). Carbon inventory methods and carbon mitigation potentials of forests in Europe: a short review of recent progress. European Journal of Forest Research, 126, 149156.

Lopes, D., (2005). Estimating net primary production in Eucalyptus globulus and Pinus pinaster ecossytems in Portugal. Ph. D. Thesis, Kingston: Kingston University.

Satterthwaite, F. E. (1946). An approximate distribution of estimates of variance components. Biometrics, 2, 110-114.

Somogyi Z., Teobaldelli, M., Federici, S., Matteucci, G., Pagliari, V., Grassi, G., \& Seufert, G. (2008). Allometric biomass and carbon factors database. Journal of Biogeosciences and Forestry, 1, $107-$ 113.

Ter-Mikaelian, M., \& Korzukhin, M. D. (1997). Biomass equations for sixty-five North American tree species. Forest Ecology and Management, 97, 1-24.

Wood density Database (2011). URL (last checked 29 June 2011) http://www.worldagroforestrycentre.org/sea/Products/AFDbases/wd/ index.htm

Zianis, D., Muukkonen, P., Mäkipää, R. \& Mencuccini, M. (2005). Biomass and stem volume equations for tree species in Europe. Silva Fennica Monographs, 4, 63. 